\title{
Towards a realistic in-vitro experience of epidural Tuohy needle insertion
}

\author{
Neil Vaughan ${ }^{1}$ and Venketesh N. Dubey ${ }^{1}$ \\ Michael Y. K. Wee ${ }^{2}$ and Richard Isaacs ${ }^{2}$ \\ ${ }^{1}$ School of Design, Engineering and Computing, Bournemouth University, Fern Barrow, Poole, BH12 5BB, UK \\ ${ }^{2}$ Department of Anaesthesia, Poole Hospital NHS Foundation Trust, Longfleet Road, Poole, BH15 2JB, UK
}

\begin{abstract}
The amount of pressure exerted on the syringe and the depth of needle insertion are the two key factors for successfully carrying out epidural procedure. The force feedback from the syringe plunger is helpful in judging the loss of pressure and the depth of the needle insertion is crucial in identifying when the needle is precisely placed in the epidural space. This paper presents the development of two novel wireless devices to measure these parameters to precisely guide the needle placement in the epidural space. These techniques can be directly used on patients or implemented in a simulator for improving the safety of procedure. A pilot trial has been conducted to collect depth and pressure data with the devices on a porcine cadaver. These measurements are then combined to accurately configure a haptic device for creating a realistic in-vitro experience of epidural needle insertion.
\end{abstract}

KEYWORDS: Epidural, Tuohy needle, insertion force, depth measurement, anaesthesia, haptic.

\section{INTRODUCTION}

Epidural is commonly used as a form of pain relief during childbirth, for the treatment of chronic back pain or as a means to provide anaesthesia or analgesia during specific operations. The procedure consists of a long Tuohy needle being carefully inserted in the lumbar spine through layers of skin, tissue and ligament into the epidural space. Monitoring pressure and the needle depth during an epidural insertion is crucial because it indicates when the needle is placed precisely into the epidural space. If the needle is advanced too far it will puncture the dural sac and cause leakage of cerebrospinal fluid. Post dural puncture headaches may result and can be extremely disabling for the patient. Other risks include bleeding or nerve damage which may rarely lead to paralysis. If the needle is not within the epidural space, the pain relief may be ineffective or inadequate due to improper placement of the catheter. Data from the Royal College of Anaesthetists revealed that permanent harm from epidurals ranged from 3.1 to 6.1 in 100,000 per year [1] and a recent review of claims handled by the National Health Service Litigation Authority (NHSLA) between 1995-2007 reported that 366 of 841 cases (44\%) were related to regional anaesthesia and of these, half arose in the obstetric setting [2]. This not only highlights the potential morbidity from epidurals but also the substantial cost burden to the national health services.

The procedure of inserting an epidural needle requires great skill and prolonged training, and can be challenging even for experienced anaesthetists. The operator is required to perceive an anatomical image of the bony alignments and the various tissue layers the needle tip is passing through by feeling the resistances on the needle from skin, through to subcutaneous fat, supraspinous and interspinous ligaments, ligamentum flavum and then to the epidural space as shown in Figure 1. Epidural needle insertion is essentially a 'blind' procedure, but utilises a well-known technique referred to as "loss of resistance" (LOR). LOR essentially involves identification of the epidural space by the loss of pressure as the epidural needle travels through the various ligaments and potential spaces of the lumbar vertebral column [3]. There are many risks involved with the epidural procedure due to its complexity. Since the actual insertion is 'blind' and the end-point cannot be visualized, the anaesthetist has only his/her perception from sensing and feeling of the needle resistance 
through the force stimuli in the fingertips and joints. There can be errors of judgment as to the location of the needle, exacerbated by individual patient characteristics of height, weight and body shape. To improve the safety of the procedure interspinous pressure as well as depth of needle insertion could be monitored electronically without interfering with the procedure for the loss of pressure and for the depth of needle (to a pre-determined value taken from ultrasound or MRI imaging).

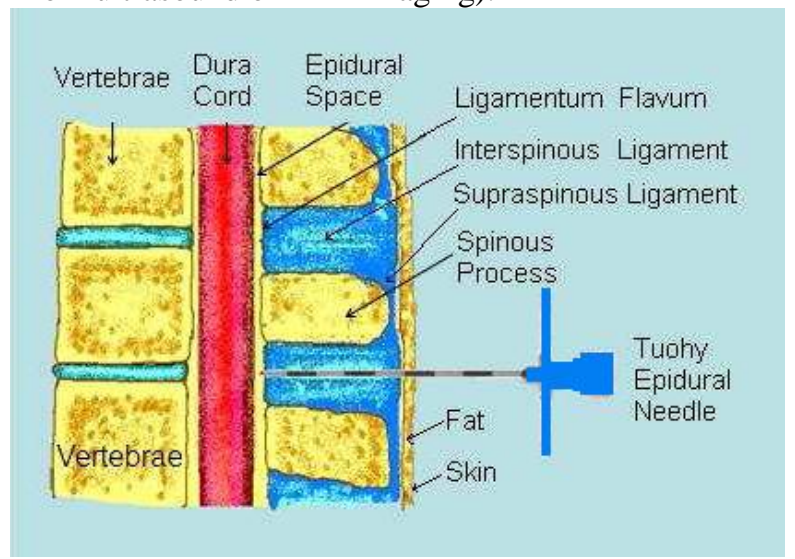

Figure 1. Anatomical structure of lumber spine with epidural needle insertion

Basic epidural simulators were introduced in 1980 and have been developed and improved upon since. Recently computer based simulators have enabled graphic visualisation of the needle amongst the tissues. Mediseus [4] and Episim [5] are examples of such simulators which were made commercially available, consisting of a haptic feedback device inside a portable case, which connected to a laptop with graphic display. One of the weaknesses of Mediseus and other devices which was highlighted in subsequent reports $[6,7]$ was the lack of measured data for the force feedback. This work aims to overcome this by basing force feedback on measured data.

This paper presents the design of a sterile pressure measurement device which captures the resultant pressures as the epidural needle is advanced through the tissue layers and a novel image processing technique which measures the insertion depth of the needle in real-time and transmits data wirelessly. The device has been tested on a fresh porcine specimen by experienced anaesthetists and the results are presented. Our preliminary porcine force measurements were presented in [8]. This work expands giving more results and also introduces new contributions of two wireless devices with details of their use and construction. Additionally this work introduces the new idea of image processing for length measurement, and includes further configuration of the haptic device and the analysis of resulting force feedback. The graphs describing the path of the needle through to the epidural space are used to recreate a realistic in-vitro experience of needle insertion. This could be potentially used to monitor epidural administration in the actual clinical procedure to reduce the risks of failure or to improve the safety of the procedure by providing training with accurately configured epidural simulators. This will help reduce morbidity and risks to patients.

\section{PRESSURE MEASURING SYSTEM DESIGN}

Our study is the first to use a wireless setup for interspinous pressure measurement which uses entirely sterile components. A wireless data transmitter is utilized to minimize the equipment and disruption in the labour room, which was XBee ${ }^{\mathrm{TM}}$ Pro $50 \mathrm{~mW}$ with a PCB Antenna, series 2 with 1 mile range, $2.4 \mathrm{GHz}$ frequency and $250 \mathrm{kbps}$ data rate. A computer receives the data remotely with a wireless receiver and displays a real-time graph on screen and simultaneously records the data to a file. The advantage of using a wireless transmitter is 
that the equipment is not only less cumbersome but also much less intrusive.

In obstetric practice, the LOR technique is performed with a saline-filled syringe connected directly onto the Tuohy needle [9]. Our design aims to minimize changes to this standard setup. A small standard sterile threeway tap (BD Connecta ${ }^{\mathrm{TM}}$ ) is connected between the needle and syringe (Figure 2). The tap is connected to the pressure transducer via a one meter length of saline-filled sterile manometer tubing. The transducer's electrical plug is connected by a short electrical cable to the wireless transmitter box which measures only $12 \mathrm{~cm} \times 5 \mathrm{~cm} \times 3 \mathrm{~cm}$. Transmission range tested inside the hospital building was large enough to cover the labour ward with numerous rooms and corridors. The receiver equipment is set up in a filing room located at the other end of the labour ward wing to avoid disruption. Despite several walls the transmission is very reliable and no data loss was encountered. At the remote site, a wireless receiver is connected via Universal Serial Bus (USB) to the computer. Our custom designed software displays a real-time graph on screen and writes the data to a text file for later analysis; data retrieval speed can be varied in the software.

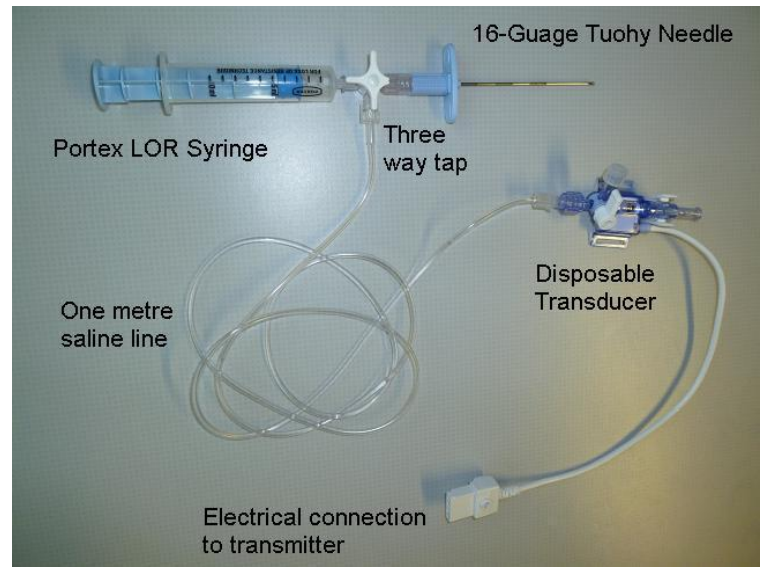

Figure 2. The disposable epidural pressure measurement system

When the anaesthetist presses on the syringe plunger, the saline inside the syringe is pressurized. The pressure waves propagate through the three-way tap, the manometer tubing and then into the disposable transducer. The transducer converts this pressure into an electrical signal $0-20 \mathrm{mV}$, which is sent to the transmitter box. The box contains a $5 \mathrm{~V}$ power supply for the transducer, an amplifier which converts the signal into a $0-5 \mathrm{~V}$ and a transmitter to relay the signal. The UTAH Medical Deltran disposable transducer is used as a pressure measurement device, supplied by Kimal Plc UK. These transducers are commonly used in hospitals to monitor systemic blood pressure and central venous pressure. Transducers produce an electrical signal based on the pressure of the liquid inside the manometer tubing. These disposable transducers are designed to have accuracy of $+/-3 \%$. In a study of accuracy of 100 disposable pressure transducers it was found that 'even the worst case transducers were twice as accurate as required by the American National Standards Institute standard, the average output was $100.03+/-0.55 \mathrm{mmHg}$, with the worst cases being 98.53 and 101.36 when $100 \mathrm{mmHg}$ was applied [10]. Standard sterile medical devices such as the disposable transducers, manometer tubing and three way tap allow a sterile set-up for each patient. All of these come packaged in a standard transducer set. The sensor signals were amplified 220 times using LM741 OP AMP integrated circuit which produce a more usable signal in the range of $0-5 \mathrm{~V} \mathrm{DC}$. The circuitry and components fit neatly inside a small plastic box along with the $5 \mathrm{~V}$ battery. A female Kimal monitor interface cable provides an easy interface for new transducers to plug into each time. 
The sensor device was calibrated by applying known weights to the plunger of the syringe. The saline-filled syringe was held vertically in place on a vice and weights were placed on the plunger at $50 \mathrm{~g}$ intervals from $0 \mathrm{~g}$ up to $1.2 \mathrm{~kg}$. The electrical outputs were recorded after amplification. It was found that voltage increased steadily up to $5 \mathrm{~V}$ with $1.2 \mathrm{~kg}$ mass, showing a linear relationship between mass and the amplified transducer voltage output. The repeatability and linearity of the device were ensured by conducting several tests under loading and unloading conditions.

Pressure can be converted into various units, in the current implementation the pressure is measured in $\mathrm{mmHg}$ or $\mathrm{kPa}$. This directly provides actual pressure measurement of saline inside the needle as applied to the continuum. From the known diameter of the plunger, force can be obtained for the load on plunger. The electrical data signal goes through a series of binary conversions before arriving at the software as a pressuretime graph. The graph shown in Figure 3 is from a needle insertion in our porcine trial, described in Section 4. The software shows only 20 seconds, so the initial pressure would have been the same as the end pressure. With wireless data transmission the graph time step is increased and can vary, although some data arrives with several values together so there is an option to interpolate the data during later analysis, decreasing the time step, which has not been done yet in this direct software view. The resulting pressure-time graph clearly shows when the LOR occurred as a drop of pressure on the graph when the needle entered the epidural space. The anaesthetists additionally performed some dural taps in porcine by inserting the needle beyond the epidural space which cut into the dura. This allowed us to capture the data from dural taps. Although this is not the goal for epidurals, the simulator needs to allow the user to insert the needle too far and produce appropriate feedback.

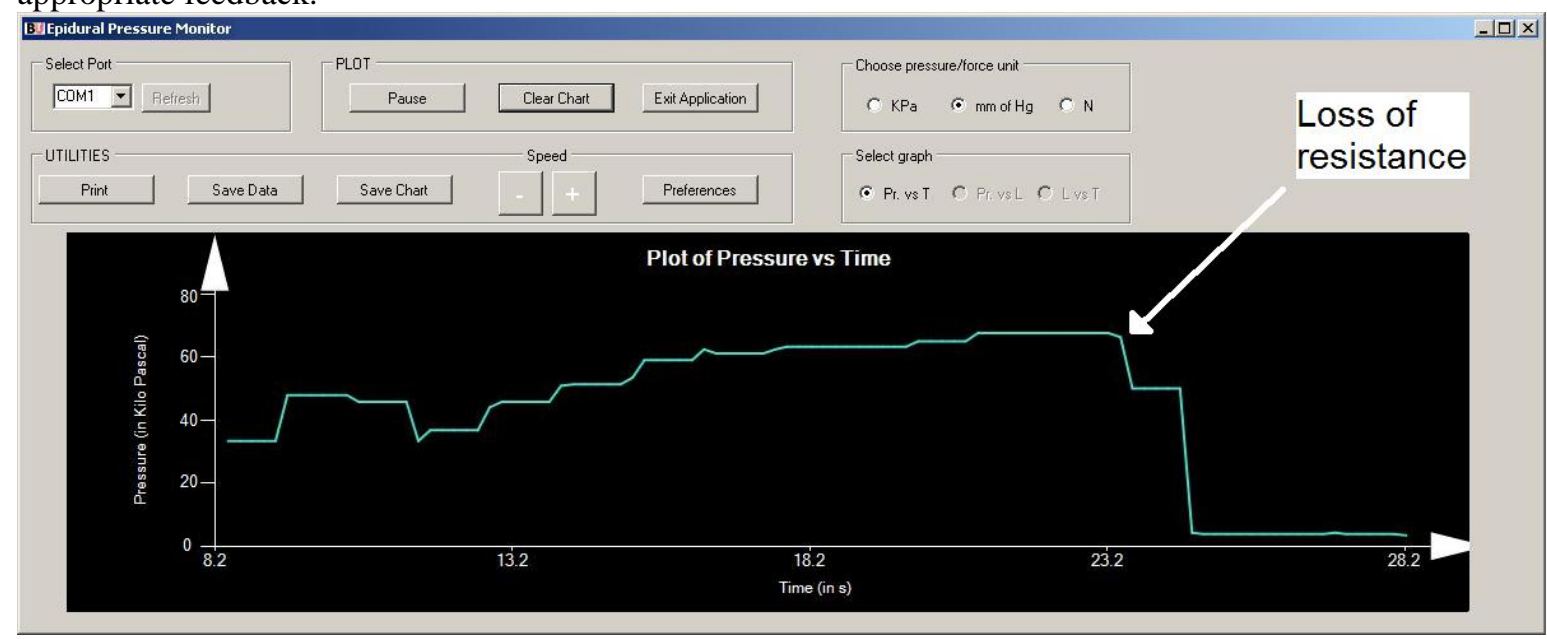

Figure 3. Screen print of the software interface to monitor and record pressure

\section{NON-CONTACT NEEDLE DEPTH MEASUREMENT}

The reason why needle depth is important is that it describes the depths at which each force occurred from the pressure transducer. This can provide information about the change of ligaments or thickness of different layers. During the epidural insertion procedure, the needle is slowly pushed through layers of tissue into the epidural space which is usually between $40-80 \mathrm{~mm}$ deep. It is useful to accurately know the needle depth because it can indicate when the epidural space has been reached for different patients. This can be accurately estimated from some non-invasive measurements such as ultrasound scanning or magnetic resonance imaging (MRI) for precise needle placement in the actual procedure. We have developed an image processing algorithm to measure the needle depth in real-time during insertion [11]. The implemented technique uses a 
single wireless camera to transmit depth data remotely to a host computer. When length and pressure data are combined, this describes which pressure occurred at which depth. The combined data can be used in the actual surgical procedure to accurately guide the needle travel or can be used to accurately configure an epidural simulator for a patient-specific training experience.

The 16 gauge Portex Tuohy needle of $80 \mathrm{~mm}$ length (Figure 4) is the most common epidural needle used in hospitals. The needle has brown and silver markings on the metallic shaft at $10 \mathrm{~mm}$ intervals which are used by the software as a reference length. The blue handle is the plastic part at the base which is held by the operator. This is used for colour detection in the current implementation. There are several problems with needle detection that makes measurement hard to accomplish: (i) the needle is a thin object with small width of only a few pixels; (ii) the needle is reflective stainless steel material which reflects colours from nearby objects, light sources and shadows; (iii) the needle is circular in cross section with different colours all around 360 degrees, the top is often illuminated while the underside is often shadowed; (iv) there are camera noise and blur from the wireless camera; (v) tilting the needle up/down causes colour change with only a small tilt of the needle; (vi) tilting towards/away from the camera causes the visible length to decrease, which may be confused with reduced length due to insertion; (vii) the needle is not the only object in the foreground, due to the operator's hands and the patient's back; (viii) lighting conditions vary from room to room; (ix) there is no time to configure the camera each time, due to the short notice given when an epidural is going to be performed and the anaesthetist cannot wait because the patient is often in pain; (x) the length is not fixed, but changes dynamically as needle is inserted. Solutions to these problems could involve controlling the environment with the use of fixed backgrounds, controlled light sources, or restricting the movement. These precautions may not be feasible since they interfere with the operator's procedure and may not be ethically permissible.

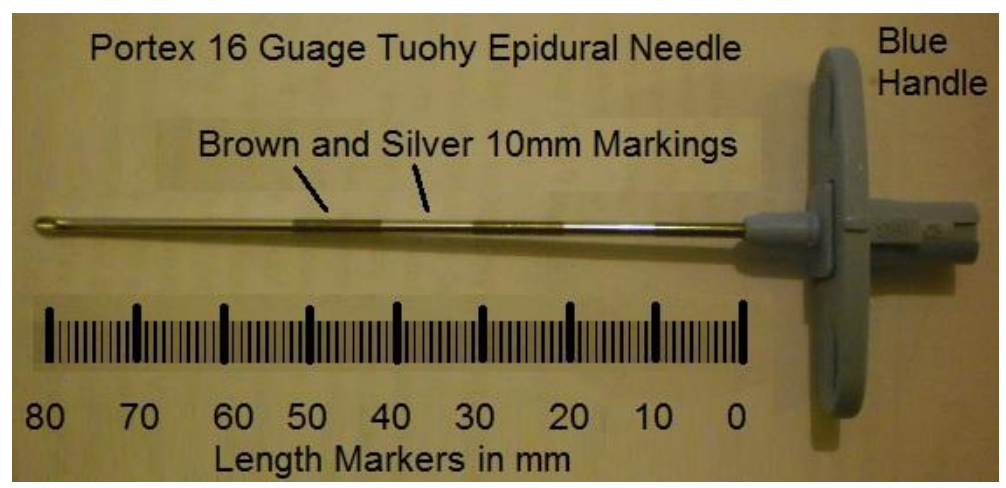

Figure 4. Properties of the Tuohy needle used in epidurals

To start the measurement, a wireless camera is placed in the operating theatre between 500 to $1000 \mathrm{~mm}$ away from the needle insertion which transmits to a computer located elsewhere in the hospital. The camera transmits a 640x480 pixel image in full colour over a 20MHz wireless link. The flow charts in Figure 5 shows the main processing steps of the image processing algorithms as implemented for depth measurement and are explained below. 


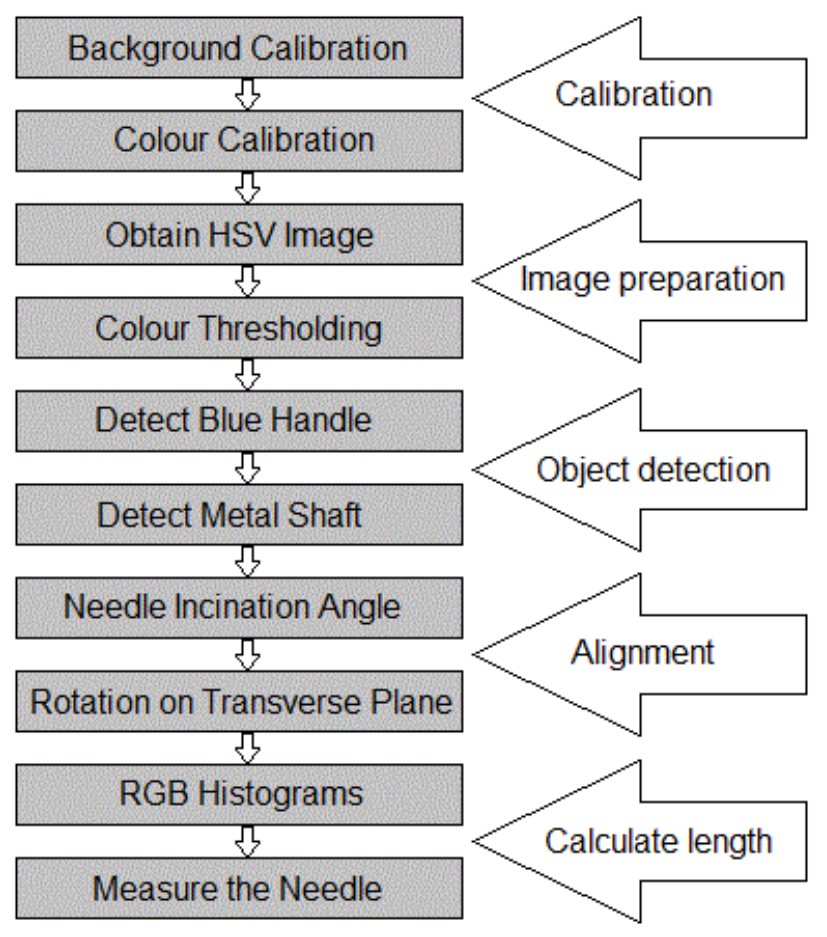

Figure 5. Image processing algorithm

\subsection{Background calibration}

Automatic calibration lasting ten seconds is necessary, which allows the algorithm to adjust to a new background. There is often short notice and urgency to administer epidurals swiftly as the patient is often in pain or requiring urgent treatment. Calibration solves issues caused by using various rooms with different lighting conditions for epidurals. To achieve background calibration, which works on any cluttered environments, the camera runs for ten seconds with no objects in the foreground. During this time, two HSV (Hue, Saturation, Value -which represents the gradation of colour within the visible spectrum of light) images are created; the minimum HSV and maximum HSV values for each pixel. These two images make up a background model. The two images are stored and used later enabling the background to be removed, identifying the foreground objects. To maximise the algorithm performance, assumptions are that movement in the image or changes in lighting during calibration and during the procedure itself are kept to a minimum to avoid false detections.

\subsection{Colour calibration}

Automatic colour calibration is done once for each new room due to changes in lighting. The blue handle is held in front of the camera and the HSV value of the blue handle is recorded. A threshold value is applied when detecting the blue handle to allow for changes in lighting. The blue handle threshold is defined as a 3D distance within HSV colour space (see Eq. 1). 


\subsection{HSV image conversion}

A new image is loaded from the camera and converted from RGB (red, green, blue) into HSV online in the software. This is a standard procedure and mathematical relationship is readily available for this conversion [12].

\subsection{Colour thresholding}

Foreground objects are identified by removing the background. This is done by comparing HSV values from every pixel in each image to the minimum HSV background values and the maximum HSV background values. If HSV is outside of the minimum-maximum range, that pixel is part of an object in the foreground. Co-ordinates of the areas containing objects are stored in an array and the rest of the algorithm is applied only to areas containing objects.

\subsection{Blue handle detection}

The blue handle is found by scanning pixels within foreground objects for RGB values which match the blue handle. A match is determined by a pixel being within the defined threshold from the recorded blue handle RGB value. To achieve accurate colour comparison, the blue handle threshold is defined as a 3D distance in the RGB colour space based on the Euclidean distance given by Eq. 1.

$$
\sqrt{(R-r)^{2}+(G-g)^{2}+(B-b)^{2}}
$$

where $R G B$ is colour 1 and $r g b$ is colour2, between which the comparison is made. Colour 1 is set to the analysed mean colour of the blue handle, which can optionally be updated by the user at run time by clicking on the blue handle pixels. Colour 2 is the colour of each pixel in the image which is being compared to colour1. This equation gives the 3D distance, equating to a spherical region in 3D colour space.

Geometrically, this gives a uniform comparison because a sphere has no extreme corner colours and the limits are all equivalent distance away from the central colour. If more or less variation is allowable in one dimension, a prolate or oblate spheroid can be used by a ratio to initial RGB values such as 0.4:0.4:0.2 before applying Eq. 1 [13]. The centre point of the blue handle is found by taking an average of the $x$ and $y$ location of all blue pixels and is stored for object tracking. The edge of the blue handle closest to the needle is stored and assumed to be the starting point of the metal shaft. The blue handle is removed from further processing.

\subsection{Metal shaft detection}

Scanning horizontally from the position of the blue handle the algorithm looks for the metal needle shaft by identifying pixels which do not match the HSV values of the background model and are arranged in a line. This allows the metal shaft to be found by identifying the line of pixels which do not match the background model. The leftmost and rightmost pixels in the metal shaft are identified and stored for tracking.

\subsection{Needle inclination angle calculation}

During epidural procedure, the needle is commonly angled along the anatomical sagittal plane up to 30 degrees to align with vertebrae. The inclination angle of the needle in the image (Figure 6) is calculated from the Cartesian coordinates of the metal needle shaft by Eq. 2 . 


$$
\theta=\tan ^{-1}\left(\frac{Y}{X}\right)
$$

where $\theta$ is needle inclination angle in degrees, $X$ and $Y$ are the differences between the leftmost and rightmost point on the metal shaft in pixels. Using $X$ and $Y$, the needle length in pixels can be found by Eq. 3 .

$$
V L=\sqrt{X^{2}+Y^{2}}
$$

where $V L$ is visible needle length in pixels i.e. the projected length to the camera.

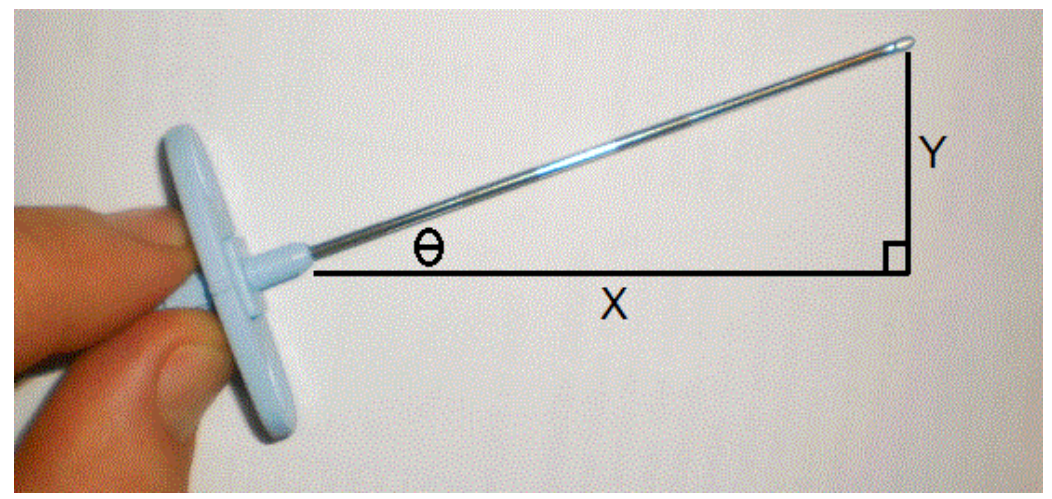

Figure 6. Cartesian coordinates of the needle

\subsection{Needle rotation of the needle on the transverse plane}

Rotation of the needle along the anatomical transverse plane is frequently used to adjust between midline and paramedian insertions. This causes tilting the needle towards / away from camera, changing the visible length of the needle which can appear similar to reduced needle length due to insertion. These two cases can be differentiated by the silver and brown markings. If all markings are present, the length change was caused by rotation on the transverse plane. The angle of rotation can be calculated by Eq. 4.

$$
\gamma=\cos ^{-1}\left(\frac{V L}{P L}\right)
$$

where $P L$ is the normal visible length in pixels (known length of the needle i.e. the silver/brown markings), $\gamma$ is needle transverse plane rotation in degrees as an offset from perpendicular to the camera. If the needle is at a fixed distance from the camera, $P L$ is a known value and this allows the equation to calculate $\gamma$. If the patient was lying on their side in lateral decubitus position, the camera could be mounted above pointing downwards.

\subsection{RGB intensity profile}

After step 3.6 a thin line of image remains of the needle. For each column in the line an average RGB value is taken. This average is used to create four separate intensity profiles for R, G, B and for the total intensity along the length of the metallic needle shaft (Figure 7). The intensity profiles identify sudden changes in colour, caused by the boundaries between $10 \mathrm{~mm}$ markings. Intensity profiles make the markings more detectable under reflective conditions. Intensity profiles are less affected by changes in lighting because separate intensity profiles are created for R, G and B to make use of whichever colour is showing the markings most clearly at that time. Occasionally individual pixels of noise in the camera cause pixels of false 
detections. These could be reduced by a longer calibration. They did not cause problems because they are ignored in subsequent steps which do not process individual pixels.

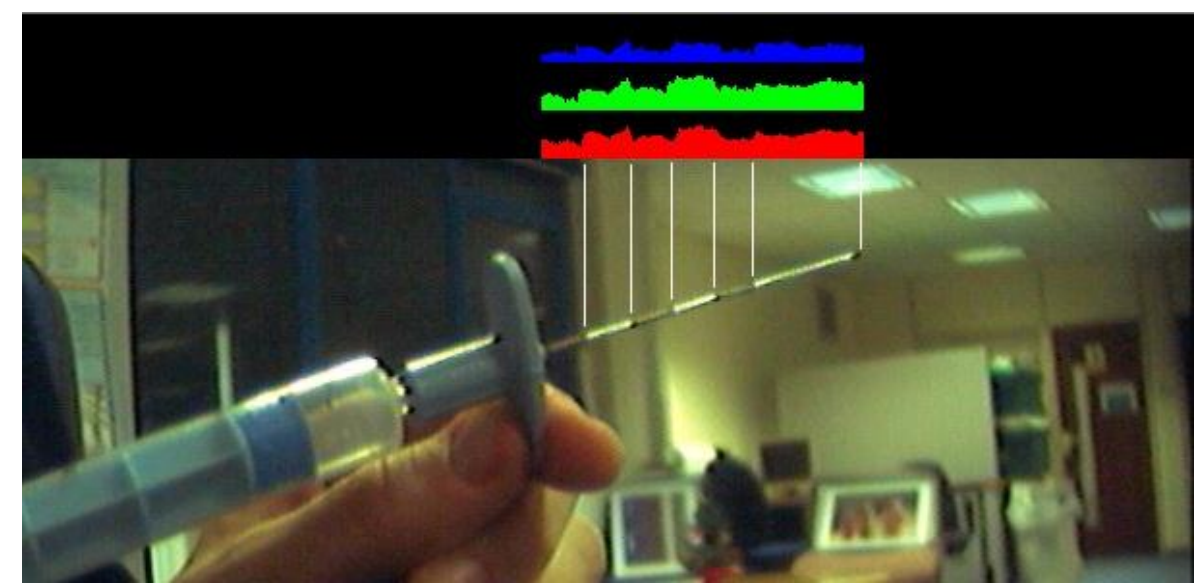

Figure 7. Intensity profile of needle drawn in real-time

Four intensity profiles (including the total intensity) are compared by the algorithm to select whichever profile shows the biggest sudden changes of value in present lighting conditions. A sudden increase or decrease indicates the edge of a silver or brown marking.

\subsection{Needle length calculation}

The intensity profiles allow the number of visible $10 \mathrm{~mm}$ markings to be counted. Also the number of pixels in each marking is counted to find how many pixels equate to one marking. This provides everything necessary to calculate needle length even when the distance between needle and camera is variable, or when vertical inclination $(\theta)$ or planar inclination $(\gamma)$ is variable. If the final marking is only partially visible, the partial length is calculated by comparing it to a full marking, using Eq. 5 .

$$
\operatorname{PartL}=F L L *\left(\frac{p}{f}\right)
$$

where PartL is length of partial marking (mm), $p$ is length of partial marking (pixels), $f$ is full length of first marking (pixels), FLL is full length of last marking (mm). Finally, lengths of all markings are combined to give full needle length by Eq. 6 .

$$
\text { Needle length }(m m)=((N-1) * 10)+(F L L *(p / f))
$$

where $N$ is number of markings. This equation gives the final value of needle length in millimetres.

\section{PILOT TRIAL FOR PRESSURE MEASUREMENT}

A porcine trial was conducted on a section of a cadaver for measurement of syringe pressure during insertion. The pig is claimed to be the most representative animal model for human spinal research. Porcine vertebrae can be a representative model when testing instrumentation techniques [14]. The porcine tissue specimen was a double loin saddle cut. The cadaver was obtained from a livestock farm within 24 hours of slaughter without being frozen or modified in any way after slaughter to preserve the integrity of the spinal tissues. The pig was a standard hybrid Large White cross Saddleback. The specimen contained the entire back in one piece, with 
the whole spine, and all tissue layers from external skin, through to the thoracic cavity. The porcine tissue was mounted vertically against a wooden support to mimic sitting position, resting upon, but not attached onto, a desk beneath as shown in Figure 8. The wooden support was fixed in place so that the cadaver would not move when pressure was applied during insertion. As time progresses the back muscles develop rigor mortis which occurs due to the cross-linking of muscle fibres. It is not known whether or not this had developed during the experiment. Internal ligaments such as ligamentum flavum and interspinous ligament should remain unaffected due to their differing composition and their interior position avoids contact with the external environment.

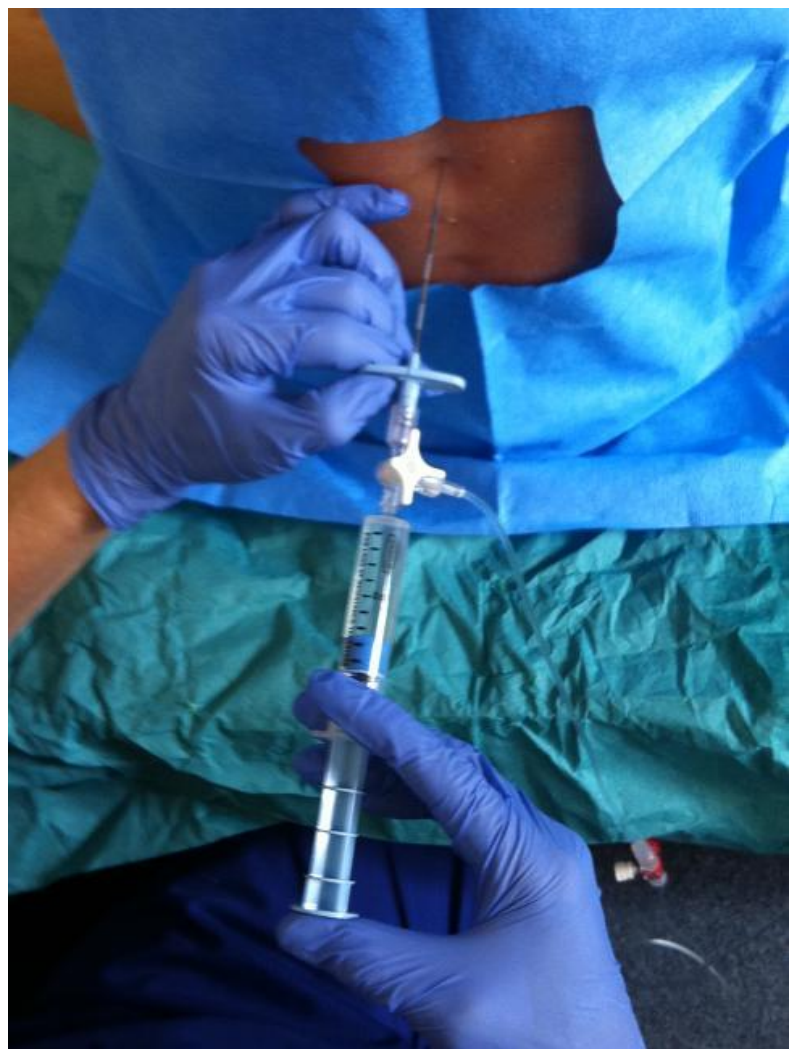

Figure 8. Porcine model for epidural insertion trial

The camera and transmitter were set up one meter away from the site which mainly focused on the needle part, therefore, needle patterns are easily discernible against the background. A saline drip bag was connected by tubing and flushed to fill the pressure transducer system. The vertical position of the transducer was compensated for the elevation difference between the transducer and the insertion point throughout to cancel any gravitational effect on the pressure readings. Epidural insertions were performed by two experienced anaesthetists. The epidural insertion point was located at L2/3 or L3/4 intervertebral levels and a Portex 16gauge Tuohy needle (Smiths Medical International Ltd, Kent, UK) was used following the midline approach [15]. Subsequently a number of different vertebral levels ranging from T12-L5 were targeted. It is believed that the thickness of tissue layers does not vary much between these vertebral levels and all layers were averaged together to produce data in Table 1 . The porcine spine was palpated to locate anatomical landmarks prior to insertion. As is the standard procedure, the Tuohy needle with its introducer stylet was penetrated into the skin, before the syringe and transducer system was attached. The recordings of pressure were then started 
and continuously recorded throughout needle insertion until after the LOR had been experienced. The majority of insertions located the epidural space in the first attempt. Data from hitting bone was recorded to analyze the effect on pressure. In some cases, the number of attempts to find the space was greater than three. Those recordings were abandoned because our study protocol which also covers the obstetric patient trial, defines that if after three attempts the space is not found, this indicates something is wrong and at that point the patient is excluded from the trial and ordinary procedure commences to reduce risk or complications such as accidental dural taps. The highest pressures were obtained when the Tuohy needle hit bone. In total 17 epidural needle insertions were performed on the porcine tissue. No more than five epidurals were attempted at a single vertebral level. The trial ceased at this point because it was decided that the presence of previous insertion attempts may begin to interfere with further readings. Table 1 shows the average value of the data collected from this trial [16]. This data compared quite well with similar previous studies $[17,18]$. To follow on from this porcine trial, a further trial with obstetric patients has begun. Ethics approval has been received from the National Research Ethics Service (NRES), reference 11/SC/0196, June 2011. The trial on human subjects could be used to further precisely simulate the needle insertion.

\begin{tabular}{|l|c|c|c|}
\hline \multicolumn{1}{|c|}{ Tissue Layer } & Tissue Thickness $(\mathrm{mm})$ & Needle Depth $(\mathrm{mm})$ & Insertion Force (N) \\
\hline Skin & 3 & 0 & 12.9 \\
\hline Subcutaneous fat & 6 & 3 & 6 \\
\hline Supraspinous ligament & 4 & 9 & 9 \\
\hline Interspinous ligament & 26 & 13 & 8 \\
\hline Ligamentum flavum & 3 & 39 & 11.1 \\
\hline Epidural space & 6 & 42 & 2.0 \\
\hline Dura & 15 & 48 & \\
\hline
\end{tabular}

Table 1. Data from porcine trial

The column for Insertion Force in Table 1 was converted from the fluid pressure over the area of the syringe barrel. Fluid pressure is constant throughout the pressure vessel so there is no time delay for the pressure to arrive at the transducer one meter away. This force is the total reaction force caused by the operator pushing with the thumb for the needle to traverse the layer, including cutting tip force, friction, static friction, tissue stiffness. Epidural space was quoted as $0 \mathrm{~N}$ because it is a potential space and once in it, the needle is already touching the dura so subsequent force will come from the dura. The measurements processed by the microcontroller have a resolution of 10 bits, giving 1024 possible values.

Figure 9 shows the shapes of 12 of the 17 porcine insertion graphs. The other 5 were not suitable for comparison because they either hit bone or were dural taps. 

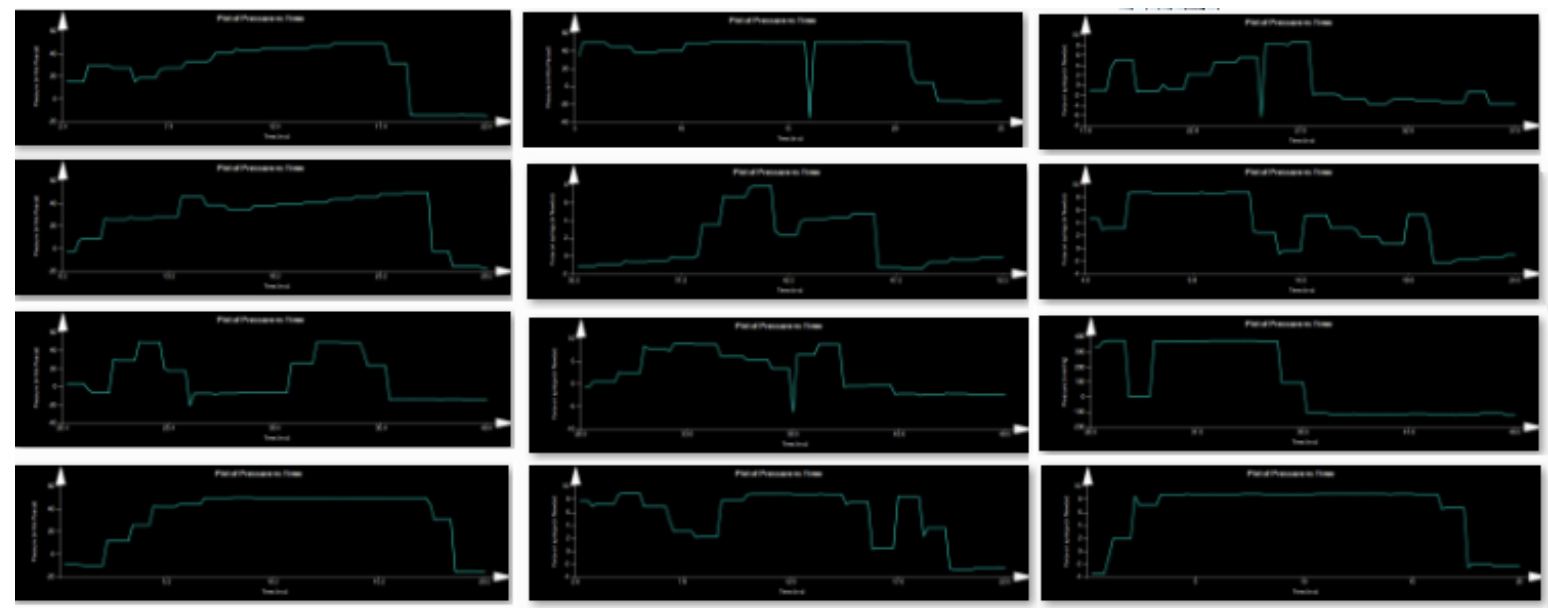

Figure 9. Needle insertion pressure graphs from the porcine trial.

\section{NEEDLE DEPTH MEASUREMENT TRIAL}

The image processing algorithm was tested during insertions. The needle was successfully detected and the depth was measured accurately in most frames. The developed software was used to draw a graph of the length in real time and write the length data to a file. Figure 10 shows length-time graph during an insertion in which the needle was slowly advanced and then rapidly withdrawn. The maximum error in all trial runs was found to be within $3 \mathrm{~mm}$, when the needle was $500 \mathrm{~mm}$ from the camera. The error of $3 \mathrm{~mm}$ was the worst case error and usually error was smaller than this. The video recordings allow certain frames to be reviewed afterwards to check the identified depths. This accuracy was high enough to ensure close approximation of depth in the majority of frames. The particular graph is chosen to show some erroneous readings at about 4 and 8 seconds, which was due to camera noise when the needle shaft was not detected properly, but all other frames were successfully measured and verified by the actual measurement. Such errors can be rectified in the software from the continuity of the graph or by ignoring sudden jumps in the data [19], just shown here to represent the extreme measurement shortfall. The total insertion took about fifteen seconds with 10 frames per second. The failure rate was 3 frames out of 150 which gave an overall $97.8 \%$ reliability during this insertion [11]. The graph currently displays length but this can be converted from length to needle depth by simply subtracting the value from $80 \mathrm{~mm}$, which is the total length of the needle. 


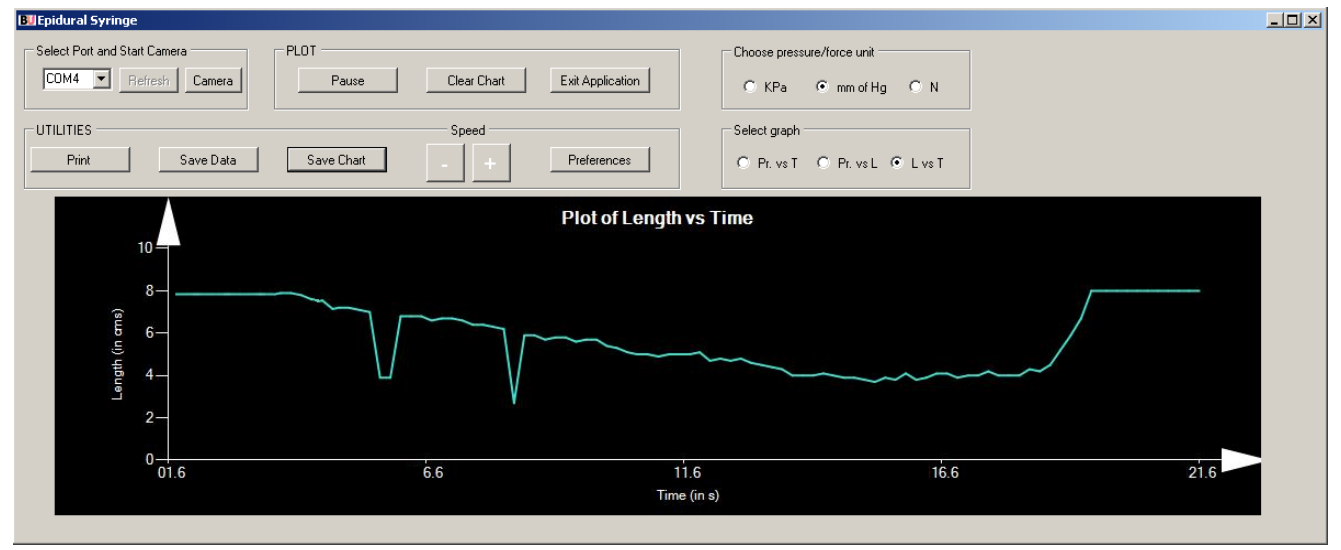

Figure 10. Plot of needle length during insertion

The distance between needle and camera can be varied between $500-1000 \mathrm{~mm}$. At distances over $150 \mathrm{~cm}$ the reliability dropped, this could be improved with a higher resolution camera. The needle can be tilted up or down to +/-30 without any effect. Tilting towards or away from the camera does not affect measurement as long as the brown and silver markings are clearly visible because marking length differentiates between length reductions caused by tilt and caused by insertion. Failures occurred on some frames, due to blur in the image, or at certain angles where markings became merged.

\section{CONFIGURATION OF A HAPTIC DEVICE ON THE MEASURED DATA}

Haptic devices have become a more popular and accepted tool for medical simulations, providing an accurate way of re-creating the feel of surgery [20,21]. The epidural insertion procedure relies almost entirely upon feeling forces on the syringe plunger and therefore epidural simulators are an ideal use for haptic technology. Haptic devices have been used in epidural simulators previously [22], although measured data was not incorporated, making it hard to assess the accuracy of the feedback forces.

A 3-DOF Novint Falcon ${ }^{\mathrm{TM}}$ haptic device was set up to move the needle in 3D, and provide force feedback to the user during insertion [23]. As the haptic stylus is moved, the needle moves on the screen and the depth of the needle tip indicates which tissue layer is being penetrated (Figure 11).

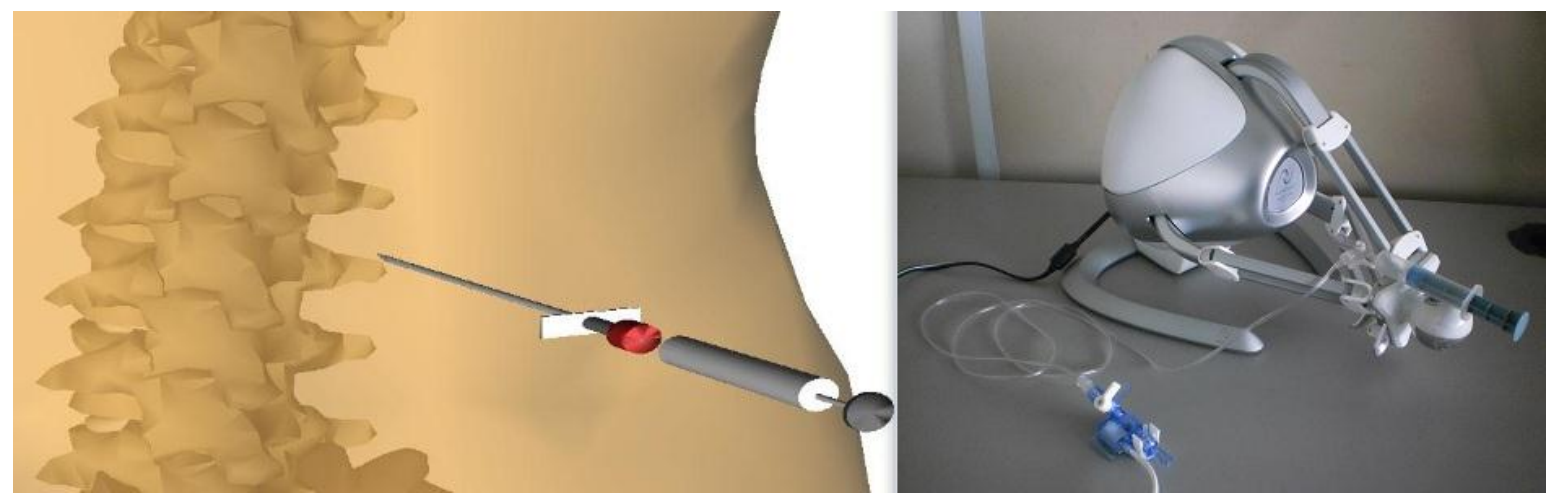

Figure 11. Haptic device configured with the measured data 
The interaction forces have been approximated to the resultant force obtained during the trial representing the force generated by the haptic device. To apply different forces to each layer, 3D vector regions were defined within the graphics model. As the needle tip enters these regions, the software identifies which tissue layer the needle is in, based on the depth data. The software uses a Look Up Table (LUT) to find the appropriate force for each layer and interpolates the data to generate that force in the haptic device. The use of LUTs to find the appropriate force for each layer to generate force in the haptic device has previously been used in the context of needle insertion [24].

The graph in Figure 12 shows the discrete forces applied by operator's thumb and the reaction forces generated by the haptic device. The reaction forces represent the resultant force on the syringe which is the sum of all resistances to insertion, equal and opposite to the applied force. For example, if a particular layer has insertion force of $4.3 \mathrm{~N}$, and the user is pressing with only $3.2 \mathrm{~N}$, then the haptic device exerts $3.2 \mathrm{~N}$, so the stylus remains stationary. Only if the user increases the force to over $4.3 \mathrm{~N}$ the stylus will move forward.

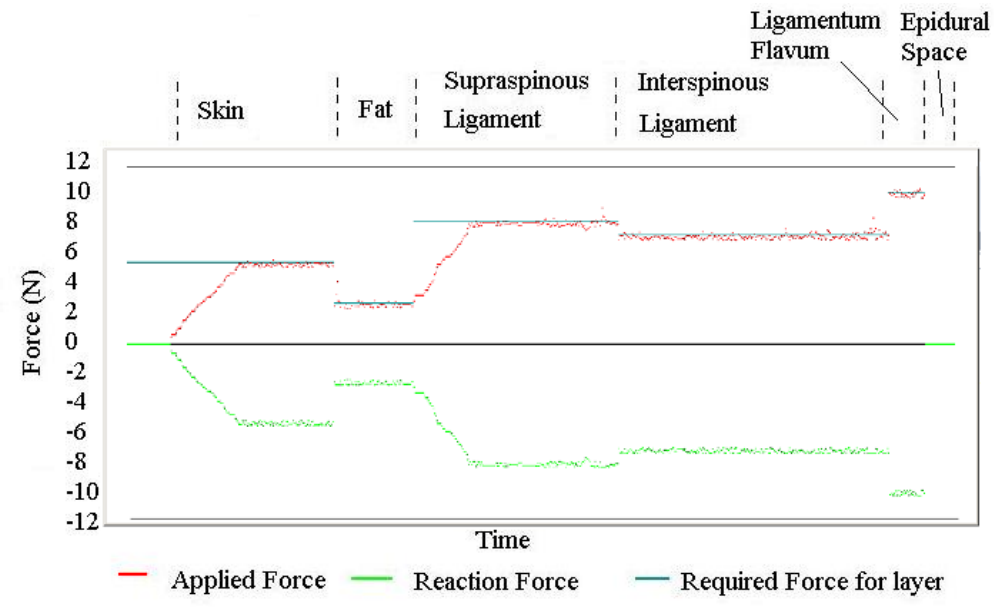

Figure 12. Haptic feedback during insertion

\section{DISCUSSION AND CONCLUSIONS}

In this paper pressure and depth measuring devices have been presented. The data for pressure and depth were successfully captured during epidural insertions. The maximum pressure and depth measurements do match closely with the results from previous related studies. The results confirm correct functioning of our devices and the ability of this setup to be further used to take measurements from epidural administration in obstetric patients.

For each needle insertion a graph was produced. The anaesthetists' feedback was that porcine tissue did feel like a close approximation to human tissue and the shape of the graphs was similar to graphs previously reported from human insertions [18]. In most cases the resulting graphs clearly show when the LOR occurred as the needle entered the epidural space (Figure 3). In some cases the needle hit bone, causing a peak on the graph [8], but with re-angulations and further advancement of the needle, the LOR was still achievable.

The developed algorithm for measuring the length of an epidural needle using the markings on its metal shaft is clinically useful as there is currently no other way to measure the needle or monitor the depth in real-time 
during insertion. This could potentially be useful for precise location of Tuohy needle during epidurals to improve safety of the procedure. The image processing method is more ethically safe and less intrusive than using physical measuring devices which touch the needle [18]. It also ensures sterility which is essential for the prevention of infection during epidural insertion. During the ten second algorithm calibration, it is preferable if no personnel were present in the camera image. This may become difficult in-vivo if there is urgency to treat the patient there may not be much time. After calibration, if a light is shifted or objects are moved it may cause complications for the algorithm, so should be avoided. However the calibration allows the algorithm to adapt to the background, enabling it to be moved into whichever room is used for the procedure.

In the current implementation the device does not allow angling of the needle. This is often required in-vivo since inserting at an angle increases the chances of the needle correctly passing into the space between vertebrae. This cannot be currently achieved with the current 3-DOF haptic device but to support this, a 6DOF haptic device would be required to allow rotation of the needle at any angles.

A further study with labouring women has now begun fould be useful for developing a realistic force feedback epidural simulator for the best possible training platform to anaesthetists. The specific ally we aim is to measure pressure differences between various patients, which will allow a generic patient-specific epidural simulator to be developed, which is currently not available. This will offer a high fidelity simulator based on clinically measured data for a realistic in-vitro experience before attempting the procedure on the actual patients thus improving the safety of the procedure and reducing the morbidity.

\section{ACKNOWLEDGEMENT}

The research was jointly funded by Bournemouth University and Poole Hospital NHS Foundation Trust.

\section{REFERENCES}

1. Cook TM, Counsell D and Wildsmith JAW. Major complications of central neuraxial block- a report on the Third National Audit of The Royal College of Anaesthetists. British Journal of Anaesthesia, 2009, 102, pp. 179-190.

2. Szypula K, Ashpole KJ, Bogod D. et al. Litigation related to regional anaesthesia: an analysis of claims against the NHS in England 1995-2007. Anaesthesia, 2010, 65, pp. 443-52.

3. Dogliotti AM. Research and clinical observations on spinal anesthesia: With special reference to the peridural technique. Anesthesia and Analgesia, 1933, 12(2), pp. 59-65.

4. Mayooran Z, Watterson L, Withers P, Line J, Arnett W, Horley R. Mediseus epidural: full-procedure training simulator for epidural analgesia in labour. Proc. SimTecT Healthcare Simulation Conference 2006.

5. Yantric Inc., EpiSim, http://www.yantric.com/products.html\#episim (accessed 12/04/2012).

6. Elks KN, Riley RH. The Mediseus epidural simulator - does it replicate real patients. Anaesth Intensive Care 2007, 35, pp. 818.

7. Lee RA, van Zundert TC, van Koesveld JJ, van Zundert AA, Stolker RJ, Wieringa PA, Gatt SP. Evaluation of the Mediseus epidural simulator. Anaesth Intensive Care, 2012, 40(2), pp. 311-8.

8. Vaughan N, Dubey VN, Wee MYK and Isaacs R. Measuring Tuohy needle insertion force on a porcine spine. IEEE 6th International Conference on Bioinformatics and Biomedical Engineering, 2012, Shanghai, China.

9. Tuohy EB. Continuous spinal anesthesia: its usefulness and technique involved. Anaesthesiology, 1944, 5, pp. 142-148. 
10. Gardner R. Accuracy and reliability of disposable pressure transducers coupled with modern pressure monitors. Critical Care Medicine 1996, 24(5), pp. 879-82.

11. Vaughan N, Dubey VN, Wee MYK and Isaacs R. Epidural needle length measurement by video processing. The IET Image Processing Conference, 2012, 3-4 July, London.

12. Agoston MK. Computer Graphics and geometric modeling: implementation and algorithms, London, Springer, 2005, pp. 300-306.

13. Paschos G. Perceptually uniform color spaces for color texture analysis: an empirical evaluation. IEEE Transaction on Image Processing, 2001, 10, pp. 932-937.

14. Busscher I, Ploegmakers JJ, Verkerke GJ and Veldhuizen AG. Comparative anatomical dimensions of the complete human and porcine spine. European Spine Journal, 2010, 19, pp. 1104-1114.

15. Carvalho JCA. Ultrasound-facilitated epidurals and spinals in obstetrics. Anesthesiology Clinics, 2008, 26, pp. $145-158$.

16. Vaughan N, Dubey VN, Wee MYK and Isaacs R. Haptic interface on measured data for epidural simulation. ASME International Design Engineering Technical Conferences \& Computers and Information in Engineering Conference, DETC2012-70891, August 12-15, 2012, Chicago, IL, USA.

17. Holton LL. Force models for needle insertion created from measured needle puncture data. Studies in Health Technology and Informatics, 2001, 81, pp. 180-186.

18. Tran D, Hor K, Kamani A, Lessoway V and Rohling R. Instrumentation of the loss-of-resistance technique for epidural needle insertion, IEEE Transactions on Bio-Medical Engineering, 2009, 56(3), pp. 820-827.

19. Grewal GS, Dubey VN and Claremont DJ. Isochromatic demodulation by fringe scanning, Strain, 2006, 42(4), pp. 273-281.

20. Coles TR, Meglan D and John NW. The role of haptics in medical training simulators: A survey of the state of the art. IEEE Transactions on Haptics, 2011, 4(1), pp. 51-66.

21. Halvorsen FH, Elle OJ and Fosse E. Simulators in surgery. Minimally Invasive Therapy and Allied Technologies, 2005, 14(4), pp. 214-223.

22. Dang T, Annaswamy TM and Srinivasan MA. Development and evaluation of an epidural injection simulator with force feedback for medical training, Studies in Health Technology and Informatics, 2001, 81, pp. 97-102.

23. Vaughan N, Dubey VN, Wee MYK and Isaacs R. Advanced epidural simulator with 3D flexible spine and haptic interface. ASME Design of Medical Devices Conference, DMD2012-6837, 10-12 April 2012, Minneapolis, MN, USA.

24. F.P. Vidal, N.W. John, A.E.Healey, D.A. Gould, "Simulation of Ultrasound Guided Needle Puncture using Patient Specific Data with 3D Textures and Volume Haptics", Computer Animation and Virtual Worlds. Vol 19, Issue 2, pp111-127, 2008, Online ISSN: 1546-427X, Print ISSN: 1546-4261. DOI: 10.1002/cav.217. 


\section{List of Figure Caption}

Figure 1. Anatomical structure of lumber spine with epidural needle insertion

Figure 2. The disposable epidural pressure measurement system

Figure 3. Screen print of the software interface to monitor and record pressure

Figure 4. Properties of the Tuohy needle used in epidurals

Figure 5. Image processing algorithm

Figure 6. Porcine model for epidural insertion trial

Figure 7. Cartesian coordinates of the needle

Figure 8. Intensity profile of needle drawn in real-time

Figure 9. Needle insertion pressure graphs from the porcine trial

Figure 10. Plot of needle length during insertion

Figure 12. Haptic device configured with the measured data

Figure 13. Haptic feedback during insertion 